\title{
Multi-Dimensional Space-Time Multilevel Codes
}

\author{
Philippa A. Martin, Member, IEEE, David M. Rankin, Member, IEEE, and Desmond P. Taylor, Fellow, IEEE
}

\begin{abstract}
To date there has been little work done on multilevel codes for the space-time environment. In this paper we develop multi-dimensional space-time multilevel codes (ST-MLCs). Several construction methods are proposed, including a coset code approach. The proposed space-time multilevel encoders partition a $2 N_{t}$-dimensional signalling space, which spans all $N_{t}$ transmit antennas. The multi-dimensional partitioning can be designed to reduce the complexity of detection/ decoding. We develop a spacetime multistage decoder for the proposed ST-MLCs. It allows the complexity of soft decision decoding to be significantly reduced compared to a single level approach. In addition, significant performance gains over a single level approach are obtained.
\end{abstract}

Index Terms-Space-time coding, multilevel coding, coset coding, multistage decoding, LDPC codes.

\section{INTRODUCTION}

$\mathbf{T}$ O DATE there has been little research into using multilevel codes $(M L C S)$ or coset codes in a space-time (multiple transmit antenna) environment. To our knowledge, the first reference to multilevel space-time codes (STCS) is Section III-H in [1]. In [1] the signal constellation ( $M$-QAM or $M$-PSK) ${ }^{1}$ is partitioned into $P$ levels using set partitioning [2] (and the idea of cosets), where each level uses a different encoder. The example in [1] uses binary partitions and simple length $N_{t}$ block codes on each level, where $N_{t}$ is the number of transmit antennas.

The space-time MLC presented in [3], [4] uses $P=$ $N_{t} \log _{2}(M)$ levels, where each level uses a binary partition of the 2-dimensional (2-D) constellation ( $M$-PSK or $M$-QAM) for a single transmit antenna [3], [4]. Thus, each component code in the MLC spans only a single transmit antenna, although it is mentioned that a multi-dimensional (multi-D) mapping could be used [3]. The design strategy is based on capacity arguments [3] and equivalent channel capacities are used to specify the desired component $\operatorname{code}^{2}$ rates [3], [4]. The design approach results in independent signaling on each transmit antenna.

Hybrid coded modulation (HCM) for the space-time environment is also proposed in [3]. It uses $N_{t}$ levels, where each level uses a bit interleaved coded modulation (BICM) scheme over $\log _{2}(M)$ bits and a single transmit antenna. Thus,

Manuscript received January 20, 2005; revised August 2, 2005; accepted August 31, 2005. The associate editor coordinating the review of this paper and approving it for publication was A. Conti. This research was supported in part by the New Zealand Foundation for Research, Science and Technology.

The authors are with the Department of Electrical and Computer Engineering, University of Canterbury, Christchurch 8020, New Zealand (email: philippa_martin@ieee.org, dave_rankin@ieee.org, taylor@elec.canterbury.ac.nz).

Digital Object Identifier 10.1109/TWC.2006.05045.

${ }^{1} M$-ary quadrature amplitude modulation (M-QAM) and $M$-ary phase shift keying (M-PSK).

${ }^{2}$ Binary Turbo codes of length 8000 are used as component codes. each antenna uses a separate encoder and bit interleaver. It is similar to V-BLAST in some cases [3]. HCM provides similar performance to the MLCs presented in [3], but is simpler due to using fewer levels $\left(N_{t}\right.$ rather than $N_{t} \log _{2}(M)$ ) [3]. However, MLCs do not have to use binary partitions [5], as in [3], and so can also have fewer levels. Also bit interleaving can be added to any level of the MLC [6]. Unfortunately, the decoding algorithms used for the HCM and MLC schemes are not explained in [3]. However, they do state that a multistage decoder (MSD) is used and that it corresponds to successive interference cancellation in the case of HCM [3].

In [7] (and several similar papers by the authors of [7]) MLCs are concatenated with orthogonal space-time block codes $($ OSTBCs). They consider various partitioning schemes, including Ungerboeck set partitioning, mixed partitioning and block partitioning.

In this paper we design MLCs using multi-D partitioning of a $2 N_{t^{-}}$-dimensional $\left(2 N_{t}-D\right)$ real constellation or a $N_{t^{-}}$ D complex constellation. Binary and non-binary partitions can be used on each level. We will describe several $2 N_{t}$-D partitioning strategies, including a coset code approach based on [8], [9] and a multi-D partitioning approach based on [10], which we call partitioning by types. Several alternative partitioning approaches are also outlined. The multi-D partitioning allows each component code in the MLC to span all $N_{t}$ transmit antennas ${ }^{3}$. Furthermore, no additional STC is used; instead we directly design space-time MLCs (ST-MLCs). Finally, we develop a space-time multistage decoder (ST-MSD) for the proposed ST-MLCs. By carefully designing the multi-D partitioning in the ST-MLC we can reduce the computational complexity of the ST-MSD.

We describe the channels and basic communication system considered in Section II. The proposed ST-MLCs and ST-MSD are described in Section III, followed by detailed examples in Section IV. Simulation results are given in Section V and conclusions are drawn in Section VI.

\section{SySTEM DESCRIPTION}

We consider space-time systems with $N_{t}$ transmit antennas and $N_{r}$ receive antennas. The $\left(1 \times N_{r}\right)$ received vector at time $t$ can be written as

$$
\boldsymbol{r}^{t}=\boldsymbol{s}^{t} \boldsymbol{H}^{t}+\boldsymbol{n}^{t}
$$

where $s^{t}$ is the $\left(1 \times N_{t}\right)$ transmitted vector at time $t$ and $\boldsymbol{n}^{t}$ is the $\left(1 \times N_{r}\right)$ additive white Gaussian noise (AWGN) vector at time $t$. The complex $N_{t} \times N_{r}$ channel matrix at time $t$ is denoted $\boldsymbol{H}^{t}$, where the element representing the subchannel from the $i^{t h}$ transmit antenna to the $j^{\text {th }}$ receive

\footnotetext{
${ }^{3}$ This means we do not use independent signaling from each transmit antenna.
} 
antenna is denoted $h_{i, j}^{t}$. We assume that all transmit to receive antenna sub-channels are independent and that we have ideal channel state information (CSI) at the receiver, but none at the transmitter.

The 1-D noise variance is defined as ${ }^{4}$ [12], [13], [14]

$$
\sigma_{n}^{2}=\frac{N_{t} E_{s}}{2 \log _{2}(M) \mathcal{R}_{\text {ecc }}^{\text {tot }} 10^{0.1 S N R}}
$$

where $S N R$ is the average signal to noise ratio $\left(E_{b} / N_{0}\right)$ in decibels $(d B), E_{s}$ is the average energy of a 2-D constellation point, $E_{b}$ is the average energy per data bit and $N_{0}$ is the 2 -sided noise spectral density. The total rate of the error correction coding (over all levels) is defined as ${ }^{5}$

$$
\mathcal{R}_{\text {ecc }}^{\text {tot }}=\frac{\sum_{p=1}^{P} k_{p} \log _{2}\left(q_{p}\right)}{\sum_{p=1}^{P} n_{p} \log _{2}\left(q_{p}\right)},
$$

where $k_{p}$ and $n_{p}$ are the number of $G F\left(q_{p}\right)$ data symbols and encoded symbols in the level $p$ component code, respectively. The rate of the ST-MLC is then defined as $\mathcal{R}_{s t c}=\mathcal{R}_{\text {ecc }}^{\text {tot }} N_{t}$, giving the number of 2-D data constellation points transmitted per time slot. Note that this allows for overall STC rates significantly greater than one, leading to increased spectral efficiency over traditional space-time block codes.

We consider two flat Rayleigh fading channel models. We consider a correlated fading channel, where the speed of the fading is defined by the normalized fade rate, $f_{D} T$, and $T$ is the symbol period. A $3^{\text {rd }}$ order Butterworth filter with cutoff frequency $f_{D}$ is used to generate the fading gains, $h_{i, j}^{t}$, for each subchannel. We also consider an independent or ideally interleaved flat Rayleigh fading channel (as in [3]). In this case, the fading channel gains change independently in each time slot and each $h_{i, j}^{t}$ is a complex Gaussian random variable with zero mean and unit variance. This approximates fast fading or a system with sufficient interleaving.

\section{ST-MLC DESIGN}

Throughout we assume $r N_{r} \geq 4$, where $r$ is the rank of the code difference matrix of the ST-MLC. In [16] it was found that when $r N_{r} \geq 4$ the minimum Euclidean distance of the STC dominates performance. Therefore, we will design the ST-MLCs for large minimum Euclidean distance in slow flat Rayleigh fading channels ${ }^{6}$.

\section{A. Partitioning and Constellations}

We begin by describing ways to design the proposed multiD ST-MLCs. During each time slot the $N_{t}$ transmit antennas collectively transmit a single $2 N_{t}$-D constellation point. The component codes in the ST-MLC jointly choose the sequence of $2 N_{t}$-D constellation points to be transmitted. Thus the partitions of the MLC span all $N_{t}$ transmit antennas and the component codes span both space (all $N_{t}$ transmit antennas) and time, creating an overall space-time code. In contrast, each component code in the MLC of [3] spans only one transmit antenna.

\footnotetext{
${ }^{4}$ This is equivalent to the definition in [11] when $N_{t}=N_{r}$

${ }^{5}$ This is a simple generalization of the usual rate definition for MLCs [15].

${ }^{6}$ In fast fading channels we need $\delta_{H} N_{r} \geq 4$ to design for large minimum Euclidean distance, where $\delta_{H}$ is the "minimum space-time symbol-wise Hamming distance" [16].
}

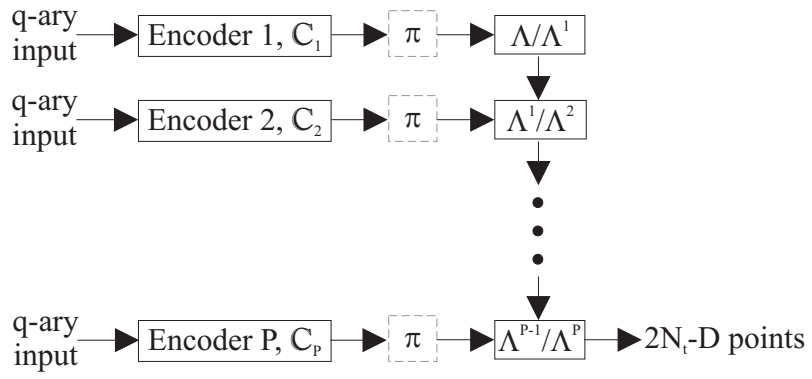

Fig. 1. Proposed $P$-level ST-MLC encoder with optional bit or symbol interleavers. Each $2 N_{t}$-D point is transmitted using $N_{t}$ transmit antennas during one time slot.

1) Coset Code Construction: We begin by developing STMLCs based on coset codes [8], [9]. We refer readers to [8], [9] for detailed information on the theory of coset codes and to [5], [15], [17], [18] for detailed information on traditional multilevel coding. We denote the partition chain of the STMLC by $\Lambda / \Lambda^{1} / \Lambda^{2} / \cdots / \Lambda^{P}$, where $P$ is the number of levels/ partitions used, $\Lambda$ is the overall $2 N_{t}$-D lattice considered and $\Lambda^{p}$ is the $p^{t h} 2 N_{t}$-D sub-lattice in the partition chain. Every point in $\Lambda^{p-1}$ can be written as a point in $\Lambda^{p}$ plus a coset representative of the partition $\Lambda^{p-1} / \Lambda^{p}$, denoted $\left[\Lambda^{p-1} / \Lambda^{p}\right]$, meaning [8]

$$
\begin{aligned}
\Lambda^{p-1} & =\Lambda^{p}+\left[\Lambda^{p-1} / \Lambda^{p}\right], \quad 1 \leq p \leq P, \\
\Lambda & =\Lambda^{P}+\left[\Lambda^{P-1} / \Lambda^{P}\right]+\cdots+\left[\Lambda / \Lambda^{1}\right] .
\end{aligned}
$$

The number of cosets within partition $\Lambda^{p-1} / \Lambda^{p}$ is denoted $\left|\Lambda^{p-1} / \Lambda^{p}\right|$ and can be different for each partition in the chain. A given ST-MLC can use both binary $\left(\left|\Lambda^{p-1} / \Lambda^{p}\right|=2\right)$ and non-binary $\left(\left|\Lambda^{p-1} / \Lambda^{p}\right|>2\right)$ partitions.

The actual constellations used by the ST-MLC are scaled, translated subsets of the lattices and sub-lattices (sphere packings) [10]. Here we constrain the 2-D components of the $2 N_{t^{-}}$ D points to be elements of $M$-QAM. Alternatively, the allowed $2 N_{t}$-D points can be carved from the lattice and sub-lattices in other ways, for example by using all points within a $2 N_{t}$-D sphere. Lattices designed for Rayleigh fading channels, such as in [19], could also be used.

The proposed $P$-level ST-MLC encoder structure is shown in Fig. 1. It includes optional bit or symbol interleaving on each level. Interleaving could also be added before the transmit antennas. The component encoder on each level selects the sequence of cosets to be transmitted. Collectively, they determine the sequence of $2 N_{t}$-D constellation points to be transmitted. An example design using the coset code approach is given in Section IV.

2) Partitioning by Types: One of the biggest issues with many non-orthogonal STCs is the computational complexity of detection/ decoding. The proposed ST-MLCs are nonorthogonal STCs. Therefore, it is beneficial to design them (constellations, partitioning and component codes) with decoding in mind. To this end we use the multi-D partitioning strategy of [10] to design the ST-MLCs and corresponding ST-MSD. This approach is based on iteratively partitioning constituent lattices or constellations of lower dimension. As a result the $2 N_{t}$-D mapping can be done as a sequence of 2-D 
mappings rather than as a single $2 N_{t}$-D mapping. This will allow us to reduce the computational complexity of detection in the ST-MSD.

This multi-D partitioning method starts by considering the 2-D constituent constellation, then higher dimensional constellations and partitions are constructed using the 2-D constellation and its partition. We begin by choosing the desired minimum squared Euclidean distance (MSED) between points in the subsets of the current level, $\delta_{p}^{2}$. Then we partition the 2$\mathrm{D}$ constellation to give the desired ${ }^{\gamma}$ MSED between points in the subsets. Consider the 16-QAM constellation in Fig. 2 (a) and a desired MSED of $4 \delta_{0}^{2}$, where $\delta_{0}^{2}$ is the MSED between 16-QAM points. A 4-way partitioning [10] can be done in two stages. First divide the constellation into subsets $A \cup D$ and $B \cup C$, which gives MSED $2 \delta_{0}^{2}$. Second divide $A \cup D$ and $B \cup C$ into $A, B, C$ and $D$, which gives MSED $4 \delta_{0}^{2}$ as desired. This gives us four subsets of points, which are each called 2-D types and are denoted $A, B, C$ and $D$. Note that this is also a four-way set partitioning [2] of 16-QAM.

\section{A B $\mid$ A B \\ C D A B A B \\ C D C D}

(a)

\begin{tabular}{ll|ll}
$\mathrm{A}$ & $\mathrm{C}$ & $\mathrm{D}$ & $\mathrm{B}$ \\
$\mathrm{C}$ & $\mathrm{A}$ & $\mathrm{B}$ & $\mathrm{D}$ \\
\hline $\mathrm{D}$ & $\mathrm{B}$ & $\mathrm{A}$ & $\mathrm{C}$ \\
$\mathrm{B}$ & $\mathrm{D}$ & $\mathrm{C}$ & $\mathrm{A}$
\end{tabular}

(b)

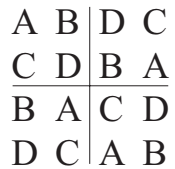

(c)
Fig. 2. Three approaches to partitioning 16-QAM into 4 subsets (A, B, C and D), each subset is called a 2-D type.

Now we can create 4-D or 8-D types by concatenating two or four 2-D types, respectively ${ }^{8}$. For example, AABC is an 8-D type, which transmits a point from $\mathrm{A}$ in the first and second 2-D subsets, then a point from $\mathrm{B}$ and $\mathrm{C}$ in the third and fourth 2-D subsets, respectively. In our case each 2-D subset is transmitted from a different transmit antenna. The MSED of each 8-D type is that of the constituent 2-D types, in this case $4 \delta_{0}^{2}$.

We call this approach, partitioning by types. Each coset on level $\mathrm{p}$ is defined by a subset of the allowed $2 N_{t}$-D types on level $p$. Each $2 N_{t}$-D type appears in only one coset. It will be considered further in Section IV, where a detailed example is given. Many other kinds of 2-D mappings can be extended to $2 N_{t}$-D using this approach. For example, the diversity-based approaches of [13], [14], [20], [21] could be used (for $r N_{r}<$ 4). There is also a lot of flexibility in choosing how the $2 N_{t}$-D types are grouped into cosets.

3) Alternative Partitioning Approaches: Another approach is to create a $2 N_{t}$-D partitioning chain by applying the algebraic method of [20], [21] to all $N_{t}$ transmit antennas. In this case a constellation carved from the $m$-D integer lattice, $Z^{m}$, is partitioned using a partitioning matrix that is designed to provide large diversity. This approach can be used to create a $2 N_{t}$-D partitioning for the ST-MLCs that is designed for large diversity. However, since we are designing for large Euclidean distance $\left(r N_{r} \geq 4\right)$, we will not consider this approach further here.

\footnotetext{
${ }^{7}$ This assumes the desired MSED is realizable.

${ }^{8}$ Higher dimensional types can be created in a similar manner [10].
}

So far we have discussed ST-MLCs using $2 N_{t}$-D partitions. However, we could also use partitions that span a subset of the dimensions/ transmit antennas as done in [3]. In [3] 2-D partitions spanning only one transmit antenna are used. But we could use two $N_{t}$-D partitions each spanning $\left(N_{t} / 2\right)$ antennas or four $\left(N_{t} / 2\right)$-D partitions each spanning $\left(N_{t} / 4\right)$ antennas. This would require a different decoding approach, possibly a combination of the approach presented in this paper and that of [3]. We call this the ST-MLC group construction method as groups of antennas are partitioned. This partitioning strategy is a generalization of [3], which contains a detailed example and is not considered further here.

\section{B. Component Codes}

As can be seen in the proposed ST-MLC encoder structure of Fig. 1, the component error correction code on each of the $P$ levels of the ST-MLC chooses a coset (or subset of $2 N_{t}-D$ points) to be transmitted. Collectively the $P$ component codes select the sequence of $2 N_{t}$-D points to be transmitted by the $N_{t}$ transmit antennas. Each $2 N_{t}$-D point is transmitted in a single time slot. Almost any coding scheme can be used as a component code in the ST-MLC [5], including concatenated codes or BICM. The ST-MLCs span $L$ time slots, where $L$ depends on the length and field size of the component codes, the number of levels and the size of the partitions. We define the length of the $q_{p}$-ary component code on level $p$ as $n_{p}=$ $L \log _{q_{p}}\left(\left|\Lambda^{p-1} / \Lambda^{p}\right|\right) q_{p}$-ary symbols.

We choose the component code rates using the capacity design rule [15], [22]. To that end we now consider the calculation of capacity for a multiple-input multiple-output $(M I M O)$ system with a fixed constellation set transmitted from each antenna [22]. Then, following the approach in [3], we will determine the capacity of each level in the partition chain. This enables us to find the ideal channel coding rates which allow the overall channel capacity to be approached [15].

The time index is dropped in this subsection as we are calculating ergodic capacity. We assume that the channel matrix, $\mathbf{H}$, is known at the receiver, but not at the transmitter. Therefore, we will not attempt to optimize the distribution of the channel input, $\mathbf{s}$. Hence the mutual information $I(\mathbf{s} ;(\mathbf{r}, \mathbf{H}))$ determines the maximum achievable rate, or capacity, through the MIMO channel [3]. We can use the chain rule to write this mutual information in the multilevel context as

$$
\begin{aligned}
I(\mathbf{s} ;(\mathbf{r}, \mathbf{H}))=I & \left(C_{1} ;(\mathbf{r}, \mathbf{H})\right) \\
& +\sum_{p=2}^{P} I\left(C_{p} ;(\mathbf{r}, \mathbf{H}) \mid C_{1}, \ldots, C_{p-1}\right),
\end{aligned}
$$

where $C_{p}$ is an index for the transmitted level- $p$ coset. Note that this is subtly different from [3]. In [3] the MLC uses binary partitions of the 2-D constellations for each transmit antenna, and thus employs a binary mapping function between the input bits and $s$. In contrast we partition the $2 N_{t}$-D constellation spanning the $N_{t}$ transmit antennas. We use binary or non-binary partitions over the $2 N_{t}$-D constellation. Thus we use a different mapping function between the input bits and $s$. This does not change the overall capacity, but simply provides a different method of approaching it. 
We now determine the capacity of each level in the STMLC, which is defined by (cf. [3])

$$
\begin{aligned}
& \mathcal{C}_{\text {cap }}^{1}=I\left(C_{1} ;(\mathbf{r}, \mathbf{H})\right), \\
& \mathcal{C}_{\text {cap }}^{p}=I\left(C_{p} ;(\mathbf{r}, \mathbf{H}) \mid C_{1}, \ldots, C_{p-1}\right), \quad 2 \leq p \leq P .
\end{aligned}
$$

This can be calculated using the techniques of [22]. Consequently, the mutual information at each level is given by [22]

$$
\begin{aligned}
& I\left(C_{p} ;(\boldsymbol{r}, \boldsymbol{H}) \mid C_{1}, \cdots, C_{p-1}\right) \\
& \quad=\sum_{\boldsymbol{s} \in\left\{\Lambda \mid C_{1}, C_{2}, \cdots, C_{p-1}\right\}} \operatorname{Pr}(\boldsymbol{s}) \int_{\mathcal{C}^{N_{r}}} f(\mathbf{r} \mid \mathbf{H}, \boldsymbol{s}) \\
& \quad \log _{2}\left(\frac{f(\mathbf{r} \mid \mathbf{H}, \boldsymbol{s})}{\sum_{\boldsymbol{s} \in\left\{\Lambda \mid C_{1}, C_{2}, \cdots, C_{p-1}\right\}} f(\mathbf{r} \mid \mathbf{H}, \boldsymbol{s}) \operatorname{Pr}(\boldsymbol{s})}\right) d \mathbf{r},
\end{aligned}
$$

where $\mathcal{C}^{N_{r}}$ is the $N_{r}$-D complex space, $\operatorname{Pr}()$ denotes the probability function and $f()$ denotes the probability density function. Monte carlo integration is used together with importance sampling to reduce the complexity of this calculation [22]. The capacity of each level is then calculated assuming previous levels transmit the zeroth $\operatorname{coset}^{9}\left(C_{1}=\cdots=\right.$ $\left.C_{p-1}=0\right)$. The $2 N_{t}$-D real constellation used by level $p$ is determined by the cosets chosen by previous levels.

The component code rate chosen by the capacity rule for level $p$ is defined as

$$
\mathcal{R}_{e c c}^{p}=\frac{\mathcal{C}_{c a p}^{p-1}-\mathcal{C}_{c a p}^{p}}{\log _{q}\left(\left|\Lambda^{p-1} / \Lambda^{p}\right|\right)} .
$$

A variety of other rate design rules could be used for the component codes of the ST-MLCs, such as those summarized in [15] (developed for the AWGN channel). The best design rule depends on several factors including the length of the component codes and the type of channel. The component codes for the ST-MLC need to perform well at the desired rates, have feasible soft decision decoding complexity ${ }^{10}$ and good distance properties. MLCs tend to require component codes with a wide range of rates, as a result different classes of component codes may be required on each level.

\section{Detection and Decoding}

Decoding a composite MLC is usually prohibitively complex and so a MSD is normally used. We now develop a STMSD for the proposed ST-MLCs. Its overall structure is shown in Fig. 3. The detection block on level $p$ generates a list of likely $2 N_{t}$-D points for that level to enable soft information to be calculated for the component error correction decoder. The lists can be found using a slightly modified version of a variety of different existing detection algorithms, including those of [11], [23], [24], [25]. In Section IV we show how the partitioning structure of the ST-MLC using partitioning by types can be exploited by the ST-MSD to reduce computational complexity.

\footnotetext{
${ }^{9}$ While the actual capacity of each level should be averaged over all coset choices, this is not expected to produce significantly different results.

${ }^{10}$ Note that STCs transmitted over a slow Rayleigh flat fading channel often require much more powerful reduced complexity decoding algorithms than for the AWGN channel [13], [14], due to having error bursts in addition to random errors. See [13], [14] for more information.
}

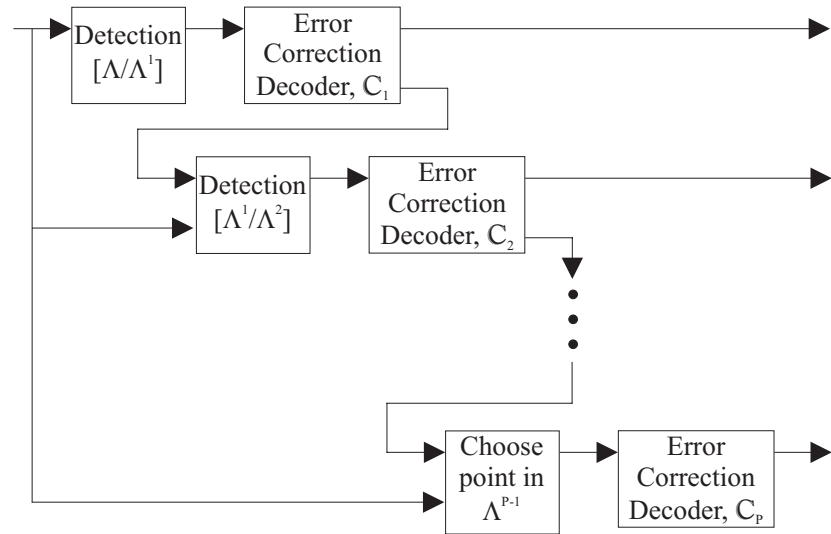

Fig. 3. Proposed ST-MSD for a $P$-level ST-MLC. If interleavers are used in the ST-MLC, then deinterleavers would need to be added to the ST-MSD. The detection boxes represent the detection and soft information calculation steps. The soft information from the detection process is passed to the error correction decoder.

We now consider the soft information calculation for each level in the ST-MSD. For simplicity of notation we assume binary component codes, however, the mathematics is easily extendable to the non-binary case. We use the terminology of cosets, however, subsets of points developed using other methods could be used.

Consider level 1, which corresponds to partition $\Lambda / \Lambda^{1}$. The coset representatives can be labelled by $m_{1}=\log _{2}\left(\left|\Lambda / \Lambda^{1}\right|\right)$ bits (or by $\log _{q_{1}}\left(\left|\Lambda / \Lambda^{1}\right|\right) q_{1}$-ary symbols). The $i^{t h}$ bit labelling the level 1 coset at time $t$ is denoted $b_{i t}^{1}$ and bits $\left(b_{1 t}^{1}, \cdots, b_{m_{1} t}^{1}\right)$ determine coset index $C_{1}$. The soft input to the binary error correction decoder corresponding to bit $b_{i t}^{1}$ can be written as

$$
\begin{aligned}
\lambda_{i t}^{1} & =\log \left(\frac{\operatorname{Pr}\left(b_{i t}^{1}=1 \mid \boldsymbol{r}^{t}, \boldsymbol{H}^{t}\right)}{\operatorname{Pr}\left(b_{i t}^{1}=0 \mid \boldsymbol{r}^{t}, \boldsymbol{H}^{t}\right)}\right) \\
& =\log \left(\frac{\sum_{\boldsymbol{s}^{1} \in\left\{\Lambda \mid b_{i t}^{1}=1\right\}} f\left(\boldsymbol{r}^{t} \mid \boldsymbol{H}^{t}, \boldsymbol{s}^{1}\right) \operatorname{Pr}\left(\boldsymbol{s}^{1}\right)}{\sum_{\boldsymbol{s}^{0} \in\left\{\Lambda \mid b_{i t}^{1}=0\right\}} f\left(\boldsymbol{r}^{t} \mid \boldsymbol{H}^{t}, \boldsymbol{s}^{0}\right) \operatorname{Pr}\left(\boldsymbol{s}^{0}\right)}\right) .
\end{aligned}
$$

Assuming all $2 N_{t}$-D constellation points $\left(\left\{\boldsymbol{s}^{1}\right\}\right.$ and $\left.\left\{\boldsymbol{s}^{0}\right\}\right)$ are equiprobable we can write

$$
\begin{aligned}
\lambda_{i t}^{1} & =\log \left(\frac{\sum_{\boldsymbol{s}^{1} \in\left\{\Lambda \mid b_{i t}^{1}=1\right\}} f\left(\boldsymbol{r}^{t} \mid \boldsymbol{H}^{t}, \boldsymbol{s}^{1}\right)}{\sum_{\boldsymbol{s}^{0} \in\left\{\Lambda \mid b_{i t}^{1}=0\right\}} f\left(\boldsymbol{r}^{t} \mid \boldsymbol{H}^{t}, \boldsymbol{s}^{0}\right)}\right) \\
& =\log \left(\frac{\sum_{\boldsymbol{s}^{1} \in\left\{\Lambda \mid b_{i t}^{1}=1\right\}} \exp \left(-\frac{1}{2 \sigma_{n}^{2}}\left\|\boldsymbol{r}^{t}-\boldsymbol{s}^{1} \boldsymbol{H}^{t}\right\|^{2}\right)}{\sum_{\boldsymbol{s}^{0} \in\left\{\Lambda \mid b_{i t}^{1}=0\right\}} \exp \left(-\frac{1}{2 \sigma_{n}^{2}}\left\|\boldsymbol{r}^{t}-\boldsymbol{s}^{0} \boldsymbol{H}^{t}\right\|^{2}\right)}\right) .
\end{aligned}
$$

The max-log MAP approximation [26] of (11) is given by

$$
\lambda_{i t}^{1} \approx \frac{1}{2 \sigma_{n}^{2}}\left(\left\|\boldsymbol{r}^{t}-\boldsymbol{s}^{0, \max } \boldsymbol{H}^{t}\right\|^{2}-\left\|\boldsymbol{r}^{t}-\boldsymbol{s}^{1, \max } \boldsymbol{H}^{t}\right\|^{2}\right),
$$

where

$$
\begin{aligned}
\boldsymbol{s}^{a, \max }= & \arg \left(\max _{\boldsymbol{s}^{a} \in\left\{\Lambda \mid b_{i t}^{1}=a\right\}}\right. \\
& \left.\left(\exp \left(-\frac{1}{2 \sigma_{n}^{2}}\left\|\boldsymbol{r}^{t}-\boldsymbol{s}^{a} \boldsymbol{H}^{t}\right\|^{2}\right)\right)\right), \quad a \in\{0,1\} .
\end{aligned}
$$


Next consider level $p$, where $p>1$. The soft input corresponding to bit $b_{i t}^{p}$ can be written as

$\lambda_{i t}^{p}=\log \left(\frac{\operatorname{Pr}\left(b_{i t}^{p}=1 \mid \boldsymbol{r}^{t}, \boldsymbol{H}^{t}, \hat{C}_{1}, \hat{C}_{2}, \cdots, \hat{C}_{p-1}\right)}{\operatorname{Pr}\left(b_{i t}^{p}=0 \mid \boldsymbol{r}, \boldsymbol{H}^{t}, \hat{C}_{1}, \hat{C}_{2}, \cdots, \hat{C}_{p-1}\right)}\right), p>1$,

where $\hat{C}_{l}$ is the index of the coset decision from level $l$. Using the same approach as for level 1 the soft input for level $p$ can be written as

$$
\begin{aligned}
\lambda_{i t}^{p}=\log & \left(\frac{\sum_{\boldsymbol{s}^{1} \in\left\{\Lambda \mid \hat{C}_{1}, \hat{C}_{2}, \cdots, \hat{C}_{p-1}, b_{i t}^{p}=1\right\}}}{\sum_{\boldsymbol{s}^{0} \in\left\{\Lambda \mid \hat{C}_{1}, \hat{C}_{2}, \cdots, \hat{C}_{p-1}, b_{i t}^{p}=0\right\}}}\right. \\
& \left.\frac{\exp \left(-\frac{1}{2 \sigma_{n}^{2}}\left\|\boldsymbol{r}^{t}-\boldsymbol{s}^{1} \boldsymbol{H}^{t}\right\|^{2}\right)}{\exp \left(-\frac{1}{2 \sigma_{n}^{2}}\left\|\boldsymbol{r}^{t}-\boldsymbol{s}^{0} \boldsymbol{H}^{t}\right\|^{2}\right)}\right)
\end{aligned}
$$

and the max-log MAP approximation as

$$
\lambda_{i t}^{p} \approx \frac{1}{2 \sigma_{n}^{2}}\left(\left\|\boldsymbol{r}^{t}-\boldsymbol{s}^{0, \max } \boldsymbol{H}^{t}\right\|^{2}-\left\|\boldsymbol{r}^{t}-\boldsymbol{s}^{1, \max } \boldsymbol{H}^{t}\right\|^{2}\right),
$$

where

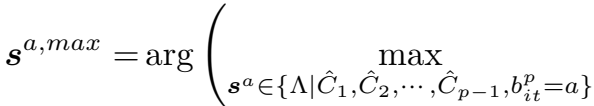

$$
\begin{aligned}
& \left.\left(\exp \left(-\frac{1}{2 \sigma_{n}^{2}}\left\|\boldsymbol{r}^{t}-\boldsymbol{s}^{a} \boldsymbol{H}^{t}\right\|^{2}\right)\right)\right), \quad a \in\{0,1\} \text {. }
\end{aligned}
$$

\section{EXAMPLE ST-MLC}

In this section we design ST-MLCs for $N_{t}=N_{r}=4$ antennas. Since $r N_{r} \geq 4$ we design for large Euclidean distance [16]. We begin with examples of partitioning using the coset code and partitioning by types approaches. Then we describe the component code design. Finally, the detection/ decoding scheme is described for the partitioning by types ST-MLC. Simulation results for the partitioning by types STMLC example are given in Section V.

\section{A. Partitioning}

In this subsection we show how equivalent ${ }^{11}$ ST-MLCs can be developed for the $Z^{8} / E_{8} / R E_{8} / 2 E_{8} / 2 R E_{8}$ partition chain [8], [9] using the coset code and partitioning by types methods. Note that these methods can create different partitions. We constrain the points in each 2-D constellation to be 16-QAM points.

1) Coset Code Approach: We first describe the coset code approach for partition chain $Z^{8} / E_{8} / R E_{8} / 2 E_{8} / 2 R E_{8}$. Note that each level uses a 16-way partition, meaning $m_{1}=m_{2}=$ $m_{3}=m_{4}=4$ in this case. This approach is based on the theory in [8], [9], from which we know that the sublattices can be defined as

$$
\begin{aligned}
E_{8} & =2 Z^{8}+(8,4,4) \\
R E_{8} & =4 Z^{8}+2(8,7,2)+(8,1,8) \\
2 E_{8} & =4 Z^{8}+2(8,4,4) \\
2 R E_{8} & =8 Z^{8}+4(8,7,2)+2(8,1,8),
\end{aligned}
$$

\footnotetext{
${ }^{11}$ In the examples, the same subsets of points are chosen on each level using both approaches.
}

where $(8,4,4),(8,7,2)$ and $(8,1,8)$ are binary Reed-Muller codes.

Data is transmitted by the ST-MLC using the coset representatives for the partitions. Therefore, we are interested in determining the coset representatives. Also since we want to transmit 16-QAM points in each 2-D subset we need to use scaled translated subsets of the lattices and sub-lattices, which we denote $\tilde{Z}^{8}, \tilde{E}_{8}, \tilde{R E_{8}}, 2 \tilde{E}_{8}$ and $2 \tilde{R} E_{8}$. The transmitted 8-D point is defined by

$$
\Lambda=2 \tilde{E}_{8}+\left[\tilde{Z}^{8} / \tilde{E}_{8}\right]+\left[\tilde{E}_{8} / \tilde{R E_{8}}\right]+\left[\tilde{R E_{8}} / 2 \tilde{E}_{8}\right],
$$

where

$$
\begin{aligned}
{\left[\tilde{Z}^{8} / \tilde{E}_{8}\right] } & =2\left[b_{1 t}^{1}, b_{2 t}^{1}, b_{3 t}^{1}, b_{4 t}^{1}\right] \boldsymbol{G}_{1}, \\
{\left[\tilde{E}_{8} / \tilde{R E_{8}}\right] } & =2\left[b_{1 t}^{2}, b_{2 t}^{2}, b_{3 t}^{2}\right] \boldsymbol{G}_{2}-4\left[0, \cdots, 0, b_{4 t}^{2}\right], \\
{\left[\tilde{R E}_{8} / \tilde{2 E}_{8}\right] } & =[2,2, \cdots, 2] b_{1 t}^{3}-4\left[b_{2 t}^{3}, b_{3 t}^{3}, b_{4 t}^{3}\right] \boldsymbol{G}_{3} .
\end{aligned}
$$

If the value of (22) in any 1-D subset is greater than 3 or smaller than -3 , then we subtract 8 or add 8 to the value, respectively, until it is in the set $\{ \pm 1, \pm 3\}$. This ensures that we transmit a point within 16-QAM that is also an appropriate lattice point (see (18)-(21) for justification on using \pm 8 ). The generator matrices are defined by

$$
\begin{gathered}
\boldsymbol{G}_{1}=\left[\begin{array}{llllllll}
0 & 0 & 0 & 0 & 0 & 0 & 1 & 1 \\
0 & 0 & 0 & 0 & 0 & 1 & 0 & 1 \\
0 & 0 & 0 & 1 & 0 & 0 & 0 & 1 \\
0 & 0 & 0 & 0 & 0 & 0 & 0 & 1
\end{array}\right], \\
\boldsymbol{G}_{2}=\left[\begin{array}{llllllll}
0 & 1 & 0 & 0 & 0 & 1 & 1 & 1 \\
0 & 0 & 1 & 0 & 1 & 1 & 1 & 0 \\
0 & 0 & 0 & 1 & 1 & 0 & 1 & 1
\end{array}\right]
\end{gathered}
$$

and $\boldsymbol{G}_{3}$ is the first three rows of $\boldsymbol{G}_{1}$. On level 4 we simply choose one of the 16 points in the translated, scaled subset of $2 E_{8}$ defined by

$$
\tilde{E}_{8}=4\left[b_{1 t}^{4}, b_{2 t}^{4}, b_{3 t}^{4}, b_{4 t}^{4}\right] \boldsymbol{G}_{4}+[1,1, \cdots, 1],
$$

where

$$
\boldsymbol{G}_{4}=\left[\begin{array}{cccccccc}
1 & 0 & 0 & 0 & 1 & 1 & 0 & 1 \\
0 & 1 & 0 & 0 & 0 & 1 & 1 & 1 \\
0 & 0 & 1 & 0 & 1 & 1 & 1 & 0 \\
0 & 0 & 0 & 1 & 1 & 0 & 1 & 1
\end{array}\right]
$$

2) Partitioning by Types Approach: Partitioning by types can also be used to give the $Z^{8} / E_{8} / R E_{8} / 2 E_{8} / 2 R E_{8}$ partition chain. It can also create non-lattice based partitions (note that [10] only considers lattice based partitions). The advantage of using partitioning by types to form this partition chain is that it leads to a reduced complexity decoder. The strategy is based on partitioning lower dimensional constituent lattices in an iterative manner [10]. As before we actually use translated, scaled subsets of the lattices and sub-lattices. For ease of explanation we will still use the term lattice and sublattice for these constellations, but now denote the partition chain as $\tilde{Z}^{8} / \tilde{E}_{8} / \tilde{R E_{8}} / \tilde{2 E_{8}} / 2 \tilde{R} E_{8}$ as before. The $2 N_{t}$-D points can be described as two consecutive 4-D points or four consecutive 2-D points [10]. As a result the overall mapping can be done as a sequence of 2-D mappings rather than as a single $2 N_{t}$-D mapping [10]. 
We shall use the 2-D types (A, B, C and D) for 16-QAM given in Fig. 2 (a) to create 8-D types. These 2-D types and resulting 8-D types provide an MSED of $4 \delta_{0}^{2}$ as shown in Section III. There are $168 D$-types which collectively define lattice $\tilde{E}_{8}$, namely

$\begin{array}{llll}C B A A & A C A B & B D A C & D A A D \\ D C B A & B B B B & A A B C & C D B D \\ B A C A & D D C B & C C C C & A B C D \\ A D D A & C A D B & D B D C & B C D D .\end{array}$

Recall that $\tilde{Z}^{8}=\tilde{E}_{8}+\left[\tilde{Z}^{8} / \tilde{E}_{8}\right]$. So, (30) gives the points in $\operatorname{coset} C_{1}=0$ of $\tilde{Z}^{8} / \tilde{E}_{8}$. The 16 8-D types for each of the other level 1 cosets can be found by adding a coset representative of $\left[\tilde{Z}^{8} / \tilde{E}_{8}\right]$, (23), to the points corresponding to the 8-D types in (30). This produces 16 new 8-D types. The partitioning of (30) provides a MSED of $4 \delta_{0}^{2}$. We note that all constellation point combinations are allowed in any 4 consecutive dimensions, but not over 8 dimensions. This can be used by the ST-MSD during detection to reduce complexity.

Now consider the level 2 partition $\tilde{E}_{8} / \tilde{R E_{8}}$. Note that $\tilde{E}_{8}=$ $\tilde{R E_{8}}+\left[\tilde{E}_{8} / \tilde{R E_{8}}\right]$. We want to partition $\tilde{E}_{8}$ to give $\tilde{R E_{8}}$ and its cosets. To do this we need to further partition the 2-D types in Fig. 2 (a) to provide a better MSED. Each of the four 2D types in Fig. 2 (a) are split into two subsets to produce the eight 2-D types $(E, F, G, H, I, J, K, L)$ shown in Fig. 4, where $A=I \cup J, B=E \cup F, C=G \cup H$ and $D=K \cup L$. There are $168 D$-types defining $\tilde{R E_{8}}$, namely

$\begin{array}{ccll}G G G G & G G H H & F F E E & F F F F \\ G H H G & G H G H & F E F E & F E E F \\ H H G G & H H H H & E F F E & E F E F \\ H G H G & H G G H & E E E E & E E F F\end{array}$

which are subsets of the 8-D types $B B B B$ and $C C C C$ in $\tilde{E}_{8}$. So, this assumes $C_{1}=0$ and $C_{2}=0$. The 8-D types for other level 1 and 2 coset combinations are created by adding the corresponding coset representatives, as defined in (23) and (24), to the points corresponding to the 8-D types in (31). This partitioning gives a MSED of $8 \delta_{0}^{2}$.
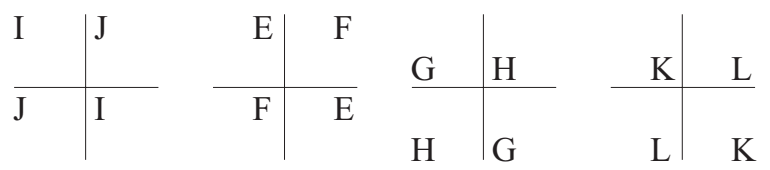

Fig. 4. The subsets (2-D types) of 16-QAM labelled A, B, C and D are each partitioned into 2 subsets (2-D types), where $A=I \cup J, B=E \cup F$, $C=G \cup H$ and $D=K \cup L$.

Now consider the level 3 partition $\tilde{R E_{8}} / 2 \tilde{E}_{8}$. Note that $\tilde{R E_{8}}=2 E_{8}+\left[\tilde{R E_{8}} / \tilde{2 E_{8}}\right]$. Now we need to partition the $2 D$-types in Fig. 4 to get individual points. This partitioning for the $2 D$-types $E, F, G$ and $H$ is shown in Fig. 5. The 16
$8 D$-types for $2 E_{8}$ are

$$
\begin{array}{cccc}
H_{2} G_{2} G_{1} H_{1} & H_{2} H_{2} H_{2} H_{2} & H_{2} G_{1} H_{1} G_{1} & H_{2} H_{1} G_{2} G_{2} \\
G_{2} H_{2} G_{2} H_{1} & G_{2} G_{2} H_{1} H_{2} & G_{2} H_{1} H_{2} G_{1} & G_{2} G_{1} G_{1} G_{2} \\
H_{1} H_{1} H_{1} H_{1} & H_{1} G_{1} G_{2} H_{2} & H_{1} H_{2} G_{1} G_{1} & H_{1} G_{2} H_{2} G_{2} \\
G_{1} G_{1} H_{2} H_{1} & G_{1} H_{1} G_{1} H_{2} & G_{1} G_{2} G_{2} G_{1} & G_{1} H_{2} H_{1} G_{2} .
\end{array}
$$

Now each 8-D type is in fact a single 8-D point. Again the 8-D types for other level 1, 2 and 3 coset combinations are created by adding the corresponding coset representatives, as defined in (23), (24) and (25) to the points in (32).

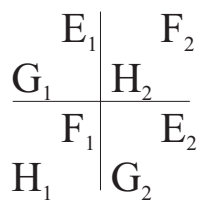

Fig. 5. Partitioning of 2-D types E, F, G and H (subsets of 16-QAM) into individual points (2-way partitions).

This is just one example of partitioning by types for $N_{t}=4$. Many other approaches could be used. For example, instead of using the initial partitioning of Fig. 2 (a) we could use those of Fig. 2 (b) or Fig. 2 (c) [20], [21], [13], [14]. Then 4-D and 8-D types could be constructed based on these 2D types. An alternate design strategy would be to design for both diversity and distance [20], [21]. However, for $r N_{r} \geq 4$ Euclidean distance has been found to dominate performance [16], [13], [14].

\section{B. Component Codes}

The component code rates chosen by the capacity rule [15], [22] are shown in Fig. 6 for the $\tilde{Z}^{8} / \tilde{E}_{8} / \tilde{R E_{8}} / \tilde{2 E_{8}} / 2 \tilde{R} E_{8}$ partition chain, where each $2 \mathrm{D}$ constellation is restricted to 16-QAM. In the present work we use binary irregular low density parity check $(L D P C)$ component codes on each level, with rates chosen using Fig. 6. LDPC codes were chosen due to their performance and simple iterative decoding algorithm (belief propagation). However, many other classes of component codes could be used.

The LDPC codes used here are designed using the near optimal degree sequences for the AWGN channel based on techniques in [27]. Specifically, the codes are designed for maximum girth using a progressive edge growth algorithm [28], which ensures an upper triangular parity check matrix for linear time encoding. The decoder uses belief propagation [29] operating in the log-domain [26]. The maximum number of decoding iterations for the LDPC code is 100 , although the LDPC decoder will terminate early if a codeword is found. A bit interleaver is used on each level after the LDPC encoder.

\section{Detection and Decoding}

The basic structure of the proposed ST-MSD is given in Fig. 3. In this subsection we describe the implementation of the STMSD used in the simulations of Section V. We first consider the detection block for each level. On each level we use a bank 


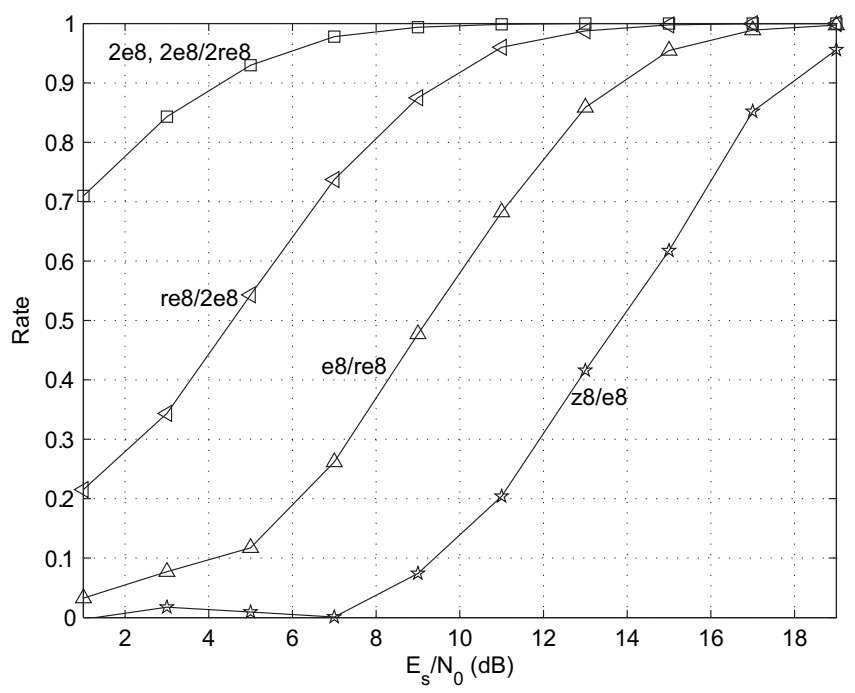

Fig. 6. Component code rates chosen using the capacity rule for the $\tilde{Z}^{8} / \tilde{E}_{8} / \tilde{R E_{8}} / 2 \tilde{E}_{8} / 2 \tilde{R E_{8}}$ partition chain, which is constrained to 16-QAM in each 2-dimensions.

of sphere decoders (SDs), with a separate SD for each coset. Each SD finds the best point in the coset, chosen according to the MSED from the received signal. Together the chosen points estimate a basic signal set [5], which is used to calculate soft information for the error correction decoder. The basic signal set consists of $\left|\Lambda^{p-1} / \Lambda^{p}\right|$ constellation points $\left(2 N_{t}-\right.$ $D$ ), namely the best point for each coset, $\left[\Lambda^{p-1} / \Lambda^{p}\right]$. Soft information is calculated using the max-log MAP approach (see (12) and (16)).

Note that, a bank of list SDs [11], [13] could also be used to create a list of $2 N_{t}$-D points for each coset. This would allow improved soft information to be calculated for each coset (see (11) and (15)), at the cost of increased computational complexity. Alternatively, a single detection process, such as a list SD (with a large list), could be used on each level. However, a very large list would often be required to obtain soft information about the less likely cosets.

The SD for a given coset considers points from the coset which lie within a radius $\sqrt{C}$ of the received signal, where $C$ is a user defined positive constant. See [23] for information on choosing $C$. If no point is found by a SD, then the distance used to calculate the soft information is set to $C$, which underestimates the true value as the real point must be outside the sphere considered by the SD. Alternatively, the value of $C$ could be increased and the SD could search for a point within the expanded sphere. For complexity reasons this is not considered here.

Partitioning by types allows us to reduce the complexity of detection. Consider level $1, \tilde{Z}^{8} / \tilde{E}_{8}$, which has the 8-D types shown in (30) for coset $C_{1}=0$. All combinations of 2-D types are allowed over two antennas, but only a single 4-D type is allowed to be transmitted from the remaining two antennas (determined by the combination of 2-D types for the other two antennas). Thus, the SD considers all possible values in four dimensions and uses the 8-D types to define the allowed values in the other four dimensions. So, a maximum of 16 points needs to be considered by the level 1 detection algorithm for two of the transmit antennas and a maximum of four points for each of the other two antennas. The SD uses lookup tables to determine the 2-D points allowed to be considered for each transmit antenna. It orders the 2-D types/ constellation points according to reliability [30]. Specifically we use a modified version ${ }^{12}$ of the SD in [13] (based on [11], [12], [30]). Likewise levels 2 and 3 can use the 8-D type information to reduce the computational complexity of the SD for each coset.

After detection and soft information calculation, the component decoder (which may be iterative internally) makes a hard decision on the transmitted coset, which is sent to the next level in the ST-MSD. This process continues until the last level, where a single $2 N_{t}$-D constellation point is chosen as shown in Fig. 3. Note that all $\left|\Lambda^{P-1} / \Lambda^{P}\right|$ remaining $2 N_{t}$-D constellation points are considered by level $P$, allowing soft information to be calculated for the level $P$ error correction decoder.

\section{RESUlts}

In this section we look at simulation results for a ST-MLC created using partitioning by types in Section IV. Many other ST-MLCs can be developed using the framework proposed in Section III. We use $N_{t}=N_{r}=4$, giving $r N_{r} \geq 4$ as required for our design [16].

We consider a 3-level ST-MLC designed based on the partition chain $\tilde{E}_{8} / \tilde{R E_{8}} / 2 \tilde{E_{8}} / 2 \tilde{R} E_{8}$ as detailed in Section IV [8], [9]. We use 3 rather than 4 levels as it is less complex and the $\tilde{Z}^{8} / \tilde{E}_{8}$ component code would have very low (near zero) rate as the overall design rate for the ST-MLC is $\mathcal{R}_{\text {ecc }}^{\text {tot }}=0.5$ (see Fig. 6) and so we do not use this level. The ST-MLC spans $L=1024$ time slots. The level 1 code for partition $\tilde{E}_{8} / \tilde{R E_{8}}$ is a $(4096,1228)$ binary LDPC code. The level 2 code for partition $\tilde{R E_{8}} / 2 E_{8}$ is a $(4096,3072)$ binary LDPC code. The level 3 code for partition $2 E_{8} / 2 \tilde{R} E_{8}$ is a $(4096,3892)$ binary LDPC code. This yields an overall code rate of $\mathcal{R}_{\text {ecc }}^{\text {tot }}=0.5$ and a STC rate of $\mathcal{R}_{\text {stc }}=2$ data (16-QAM) symbols per time slot. Therefore, 8 data bits are transmitted during each time slot. Bit interleavers are used on each level after LDPC encoding. The bit error rate (BER) and block error rate (BLER) performance of this ST-MLC for both channel models under consideration is shown in Fig. 7 and Fig. 8, respectively, where each ST-MLC codeword is a block.

For comparison purposes ${ }^{13}$ we also consider a single level ST-LDPC code. It uses a binary $(16384,8192)$ LDPC code Gray mapped to 16-QAM across the $N_{t}=4$ transmit antennas and $L=1024$ time slots. It is a direct transmission scheme [12], [11]. A bit interleaver is used between the encoder and the mapping, however, this is not necessary. The STLDPC code uses the soft output list SD of [11] with a list size of 512 (iterative decoding between the SD and error

\footnotetext{
${ }^{12}$ It is modified so that the 2-D types are restricted based on the $168-\mathrm{D}$ types associated with the coset.

${ }^{13} \mathrm{We}$ will not compare the performance of the proposed ST-MLC to those of [3] as they use only $N_{t}=2$ or 3 and $N_{r}=1$ or 2 . We design for $r N_{r} \geq 4$, meaning their designs would need to provide $r=2$ (full rank) and use $N_{r}=2$. They consider 4-PSK, whereas we are interested in 16QAM. In addition, a clear description of the decoding process is not given in [3].
} 


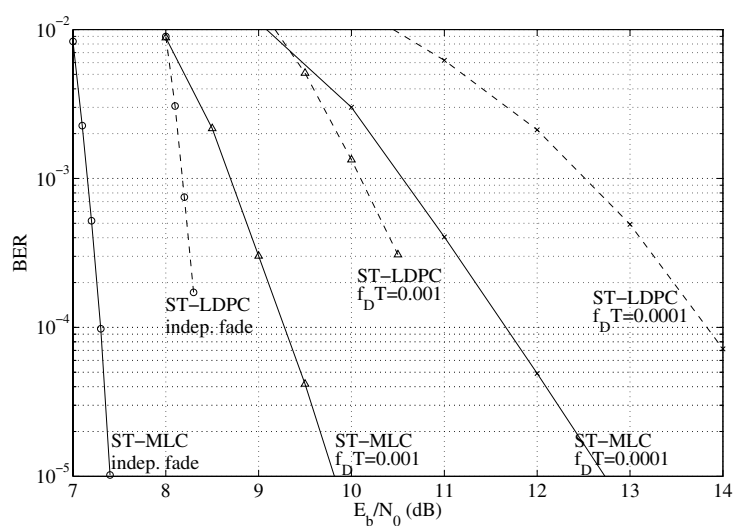

Fig. 7. BER of a rate $\mathcal{R}_{s t c}=2$ ST-MLC and a rate $\mathcal{R}_{s t c}=2$ ST-LDPC code. Each antenna transmits points from 16-QAM.

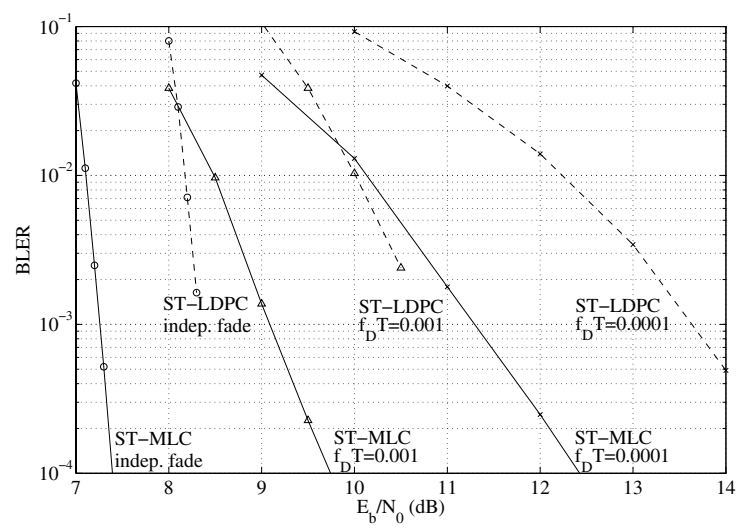

Fig. 8. BLER of a rate $\mathcal{R}_{s t c}=2$ ST-MLC and a rate $\mathcal{R}_{s t c}=2$ ST-LDPC code. Each antenna transmits points from 16-QAM.

correction decoder is not considered here). The BER and BLER performance of this code is also shown in Fig. 7 and Fig. 8, respectively. As can be seen the performance of both the ST-MLC and ST-LDPC code is worse for slower fading channels.

The decoder of [11] was used for a rate $1 / 2$ binary Turbo code with 9216 data bits (and 18432 encoded bits) in [11]. They used $N_{t}=N_{r}=4$, an independent Rayleigh flat fading channel and 16-QAM. The Turbo code obtained a BER of $10^{-5}$ at approximately ${ }^{14} 8.8 \mathrm{~dB}$. Both the ST-MLC and STLDPC code provide better performance than the Turbo code. The performance of all three approaches could be improved by using iterative decoding between the detection and decoding blocks as proposed in [11]. We believe the performance of the ST-MLC is better than that of the ST-LDPC code as it can calculate better soft information due to using $2 N_{t}$-D partitions and calculating the soft information using a basic signal set. In addition, the proposed ST-MLCs provide a good method of spreading information across space and time.

The ST-MLC uses a different detection method than the other two approaches, namely the ST-MSD. It is less complex than the approach used by the ST-LDPC code and Turbo code, as it takes advantage of the "type" partitioning structure of

\footnotetext{
${ }^{14}$ We refer readers to Fig. 7 in [11] for an exact SNR value.
}

the ST-MLC. The number of $2 N_{t}$-D points considered by the SDs during one time slot are used to compare the complexity of the ST-MLC and ST-LDPC code schemes. The ST-LDPC code results are shown in Table I at an SNR of 8.1dB. The ST-MLC used an average of 86 and a maximum of $2892 N_{t^{-}}$ D candidates at an SNR of $7.1 \mathrm{~dB}$ (this was calculated over all SDs on all levels ${ }^{15}$ ). We use list candidates to compare complexity as they account for the main complexity difference between the ST-MSD and the list SD for the ST-LDPC code ${ }^{16}$.

TABLE I

NUMBER OF $2 N_{t}$-D CANDIDATES CONSIDERED BY THE SD DURING ONE TIME SLOT FOR THE ST-LDPC CODE AT 8.1DB.

\begin{tabular}{c|c|c} 
List size & $\begin{array}{c}\text { Average number of } \\
2 N_{t} \text {-D candidates }\end{array}$ & $\begin{array}{c}\text { Maximum number of } \\
2 N_{t} \text {-D candidates }\end{array}$ \\
\hline 32 & 138 & 768 \\
64 & 274 & 1292 \\
128 & 533 & 2140 \\
256 & 1001 & 3435 \\
512 & 1826 & 5739
\end{tabular}

\section{Vi. CONCLUSiOnS}

We have developed a theoretical framework for the proposed ST-MLCs and ST-MSDs. Several construction methods for STMLCs have been presented. The proposed ST-MLCs use $2 N_{t^{-}}$ D rather than 2-D partitions. The partitioning strategy of [10] has been applied to the space-time environment, allowing the complexity of the resulting ST-MSD to be reduced. Simulation results were presented for a ST-MLC using an $E_{8}$-based partition chain and partitioning by types. It performed better than either the single level ST-LDPC code or the Turbo STC. The ST-MLC approach of this paper can easily be extended to larger number of antennas and many different signal constellations with a corresponding variety of partitioning structures.

\section{REFERENCES}

[1] V. Tarokh, N. Seshadri, and A. R. Calderbank, "Space-time codes for high data rate wireless communication: Performance criterion and code construction," IEEE Trans. Inform. Theory, vol. 44, no. 2, pp. 744-765, Mar. 1998.

[2] G. Ungerboeck, "Channel coding with multilevel / phase signals," IEEE Trans. Inform. Theory, vol. 28, no. 1, pp. 55-67, Jan. 1982.

[3] L. H.-J. Lampe, R. Schober, and R. F. H. Fischer, "Multilevel coding for multiple-antenna transmission," IEEE Trans. Wireless Commun., vol. 3, no. 1, pp. 203-208, Jan. 2004.

[4] L. H.-J. Lampe, R. F. H. Fischer, and R. Schober, "Multilevel coding for multiple-antenna transmission," in Proc. ISIT, p. 104, Lausanne, Switzerland, June 30-July 52002.

[5] G. J. Pottie and D. P. Taylor, "Multilevel codes based on partitioning," IEEE Trans. Inform. Theory, vol. 35, no. 1, pp. 87-98, Jan. 1989.

[6] D.-F. Yuan and X. Zhu, "Multiple hierarchical transmission scheme with bit interleaver over rayleigh fading channel," in Proc. VTC Fall, pp. 2439-2441, 7-11 Oct. 2001.

[7] D.-F. Yuan, F. Zhang, A.-F. Sui, and Z.-W. Li, "Concatenation of spacetime block codes and multilevel coding over rayleigh fading channels," in Proc. VTC Fall, pp. 192-196, 2001.

${ }^{15}$ Level 3 considers all $162 N_{t}$-D points, which has been included in the candidate totals.

${ }^{16}$ The simulations for Fig. 7 and Fig. 8 were slower for the ST-LDPC (list size 512) at a BER of $10^{-4}$ than for the ST-MLC at a BER of $10^{-5}$. 
[8] G. D. Forney Jr., "Coset codes - part 1: Introduction and geometrical classification," IEEE Trans. Inform. Theory, vol. 34, no. 5, pp. 11231151, Sept. 1988.

[9] —- "Coset codes - part 2: Binary lattices and related codes," IEEE Trans. Inform. Theory, vol. 34, no. 5, pp. 1152-1187, Sept. 1988.

[10] L.-F. Wei, "Trellis-coded modulation with multidimensional constellations," IEEE Trans. Inform. Theory, vol. 33, no. 4, pp. 483-501, July 1987.

[11] B. M. Hochwald and S. ten Brink, "Achieving near-capacity on a multiple-antenna channel," IEEE Trans. Commun., vol. 51, no. 3, pp. 389-399, Mar. 2003.

[12] M. O. Damen, K. Abed-Meraim, and J.-C. Belfiore, "A generalized lattice decoder for asymmetrical space-time communication architecture," in Proc. ICASSP, pp. 2581-2584, 2000.

[13] P. A. Martin and D. P. Taylor, "High-throughput error correcting spacetime block codes," IEEE Commun. Lett., vol. 8, no. 7, pp. 458-460, July 2004.

[14] — "High-throughput error correcting space-time block codes," in Proc. ISIT, p. 127, Chicago, USA, June 27 - July 22004.

[15] U. Wachsmann, R. F. H. Fischer, and J. B. Huber, "Multilevel codes: Theoretical concepts and practical design rules," IEEE Trans. Inform. Theory, vol. 45, no. 5, pp. 1361-1391, July 1999.

[16] J. Yuan, Z. Chen, B. Vucetic, and W. Firmanto, "Performance and design of space-time coding in fading channels," IEEE Trans. Commun., vol. 51, no. 12, pp. 1991-1996, Dec. 2003.

[17] A. R. Calderbank, "Multilevel codes and multistage decoding," IEEE Trans. Commun., vol. 37, no. 3, pp. 222-229, Mar. 1989.

[18] H. Imai and S. Hirakawa, "A new multilevel coding method using errorcorrecting codes," IEEE Trans. Inform. Theory, vol. 23, no. 3, pp. 371377, May 1977.

[19] J. Boutros, E. Viterbo, C. Rastello, and J. C. Belfiore, "Good lattice constellations for both rayleigh fading and gaussian channels," IEEE Trans. Inform. Theory, vol. 42, no. 2, pp. 502-518, Mar. 1996.

[20] S. Mallik and R. Koetter, "Partitioning techniques for design of multilevel codes for rayleigh fading channels," in Proc. ISIT, p. 409, Chicago, USA, June 27 - July 22004.

[21] S. Mallik, "Multilevel coding schemes for underspread fading channels," Master's thesis, University of Illinois at Urbana-Champaign, 2004.

[22] H. Zhu, Z. Shi, B. Farhang-Beroujeny, and C. Schlegel, "An efficient statistical approach for calculation of capacity of MIMO channels," in Proc. IASTED Int. Conf. Wireless Optical Commun., Bariff, Canada, July 14-16 2003.

[23] J. Boutros, N. Gresset, L. Brunel, and M. Fossorier, "Soft-input softoutput lattice sphere decoder for linear channels," in Proc. Globecom, San Francisco, CA, USA, 1-5 Dec. 2003.

[24] S. Bäro, J. Hagenauer, and M. Witzke, "Iterative detection of mimo transmission using a list-sequential (LISS) detector," in Proc. ICC 2003 .

[25] A. B. Reid, A. J. Grant, and A. P. Kind, "Low complexity list detection for high-rate multiple-antenna channels," in Proc. ISIT, p. 273, 2003

[26] P. Robertson, E. Villebrun, and P. Hoeher, "A comparison of optimal and sub-optimal MAP decoding algorithms operating in the log domain," in Proc. ICC, vol. 2, pp. 1009-1013, 1995.

[27] T. Richardson, A. Shokrollahi, and R. Urbanke, "Design of capacityapproaching irregular low-density parity check codes," IEEE Trans. Inform. Theory, vol. 47, pp. 619-637, Feb. 2001.

[28] X.-Y. Hu, E. Eleftheriou, and D.-M. Arnold, "Progressive edge growth tanner graphs," in Proc. Globecom, vol. 2, pp. 995-1001, 2001.

[29] X.-Y. Hu, E. Eleftheriou, D.-M. Arnold, and A. Dholakia, "Efficient implementations of the sum-product algorithm for decoding LDPC codes," in Proc. Globecom, vol. 2, pp. 25-29, Nov. 2001.
[30] A. M. Chan and I. Lee, "A new reduced-complexity sphere decoder for multiple antenna systems," in Proc. ICC, pp. 460-464, 2002.

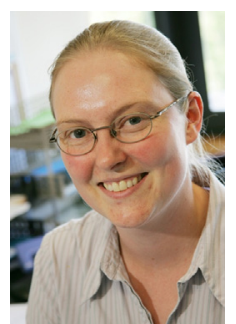

Philippa A. Martin received the B.E. (Hons. 1) and $\mathrm{Ph} . \mathrm{D}$. degrees in electrical and electronic engineering from the University of Canterbury, Christchurch, New Zealand, in 1997 and 2001, respectively.

From August 2001 to November 2004, she was a research engineer and then a FRST postdoctoral fellow in the Department of Electrical and Computer Engineering at the University of Canterbury. In 2002, she spent 5 months as a visiting researcher in the Department of Electrical Engineering at the University of Hawaii at Manoa, Honolulu, Hawaii, U.S.A.. Since December 2004 she has been a lecturer at the University of Canterbury, Christchurch, New Zealand. Her current research interests include multilevel coding, error correction coding, iterative decoding and equalization, and space-time coding and detection, in particular for wireless communications.

Dr. Martin currently serves on the Editorial Board of the IEEE Transactions on Wireless Communications.

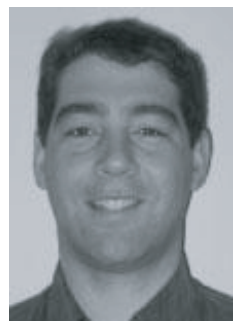

David M. Rankin received the B.E. (Hons I) and Ph.D. degrees from the University of Canterbury, Christchurch, New Zealand, in 1997 and 2001 respectively.

From 2001 to 2003 he worked as an independent researcher and embedded systems designer. From 2003 to 2005 he was a part time research engineer doing work in the area of space-time communications at the University of Canterbury while simultaneously continuing his consulting business. Currently he is working full-time in his own company working in the sensor network area. His interests include, iterative decoding, low complexity design, LDPC codes, space-time communication systems and capacity analysis of MIMO channels.

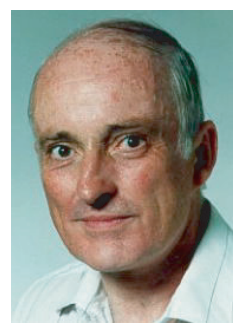

Desmond P. Taylor was born in Noranda, Quebec, Canada on July 5, 1941. He received the B.Sc.(Eng.) and M.Sc.(Eng.) degrees from Queen's University, Kingston, Ontario, Canada in 1963 and 1967 respectively, and the Ph.D. degree in 1972 in Electrical Engineering from McMaster University, Hamilton, Ontario, Canada. From July 1972 until June 1992, He was with the Communications Research Laboratory and Department of Electrical Engineering of McMaster University. In July 1992, he joined the University of Canterbury, Christchurch, New Zealand where he is now the Tait Professor of Communications. His research interests are centred on digital wireless communications systems with a primary focus on the development of robust, bandwidth-efficient modulation and coding techniques, and the development of iterative algorithms for joint equalization and decoding of the fading, dispersive channels typical of mobile radio communications. Secondary interests include problems in synchronization, multiple access and networking. He is the author or coauthor of approximately 200 published papers and holds two U.S. patents in spread spectrum communications. One paper won the S.O. Rice Award for the best Transactions paper in Communication Theory of 2001

He is a Fellow of the IEEE, a Fellow of the Royal Society of New Zealand, and a Fellow of both the Engineering Institute of Canada and the Institute of Professional Engineers of New Zealand. 